\title{
The Capitulation Problem for Certain Cyclic Quartic Number Fields
}

\author{
Mohammed First University \\ (Communicated by S. Nakano)
}

Abdelmalek AZIZI, Idriss JERRARI and Mohammed TALBI

\begin{abstract}
Let $K$ be a cyclic quartic number field such that its 2-class group is of type $(2,4), K_{2}^{(1)}$ be the Hilbert 2-class field of $K, K_{2}^{(2)}$ be the Hilbert 2-class field of $K_{2}^{(1)}$ and $G=\mathrm{Gal}\left(K_{2}^{(2)} / K\right)$ be the Galois group of $K_{2}^{(2)} / K$. Our goal is to study the capitulation problem of 2-ideal classes of $K$ and to determine the structure of $G$.
\end{abstract}

\section{Introduction}

Let $K$ be a number field of finite degree over $\mathbb{Q}$. We denote by $\mathcal{O}_{K}, E_{K}$ and $C_{K}$, the ring of integers, the unit group and the ideal class group of $K$, respectively. For a prime number $p$, let $C_{K, p}$ be the $p$-class group and $K_{p}^{(1)}$ the Hilbert $p$-class field of $K$. Further, we define $K_{p}^{(n)}$, for an integer $n \geqslant 0$, by $K_{p}^{(0)}=K$ and $K_{p}^{(n+1)}=\left(K_{p}^{(n)}\right)^{(1)}$. So we have the sequence

$$
K \subseteq K_{p}^{(1)} \subseteq \cdots \subseteq K_{p}^{(n)} \subseteq \cdots
$$

that is called the $p$-class field tower of $K$. We know that it is finite if and only if there exists a finite $p$-extension of $K$ whose $p$-class number is equal to 1 . It is well-known that if $C_{K_{p}^{(1)}, p}$ is cyclic then $C_{K_{p}^{(2)}, p}$ is trivial, implying that $K_{p}^{(2)}=K_{p}^{(3)}$ (Taussky [17]).

A fractional ideal $\mathcal{A}$ of $K$ is said to capitulate in an extension $L / K$ if $\mathcal{A \mathcal { O } _ { L }}=\alpha \mathcal{O}_{L}$ for some $\alpha \in L$.

Let $L / K$ be a cyclic unramified extension and $j=j_{L / K}: C_{K} \longrightarrow C_{L}$ the conorm of $L / K$ for ideal classes. Taussky defined:

- the extension $L / K$ is said of type $(A)$ iff $\left|\operatorname{Ker}(j) \cap N_{L / K}\left(C_{L}\right)\right|>1$;

- the extension $L / K$ is said of type $(B)$ iff $\left|\operatorname{Ker}(j) \cap N_{L / K}\left(C_{L}\right)\right|=1$.

Note that $\operatorname{Ker}(j)$ is the set of all the ideal classes of $K$ which capitulate in $L$.

Received February 25, 2015; revised July 27, 2016

Mathematics Subject Classification: 11R27 (Primary), 11R37

Key words and phrases: Cyclic quartic field, Capitulation

This work is partially supported by CNRST (PBER), Hassan II Academy of Sciences and Technology (Morocco), URAC 6 and ACSA laboratory (FSO-UMPO). 
Definition 1. Let $G$ be a group. We say that $G$ is metacyclic if there exist a normal cyclic subgroup $H$ of $G$ such that the quotient group $G / H$ is cyclic.

REMARK 1. If $G$ is a metagroup, then the commutator group $G^{\prime}$ is cyclic.

Let $K$ be a number field such that its 2-class group $C_{K, 2}$ is isomorphic to $\mathbb{Z} / 2 \mathbb{Z} \times \mathbb{Z} / 4 \mathbb{Z}$ (i.e. is of type $(2,4))$ and $G$ be the Galois group of $K_{2}^{(2)} / K$. By class field theory, $G / G^{\prime}$ is isomorphic to $\mathbb{Z} / 2 \mathbb{Z} \times \mathbb{Z} / 4 \mathbb{Z}$. Then, the following diagram shows all the unramified subextensions of $K_{2}^{(1)} / K$ :

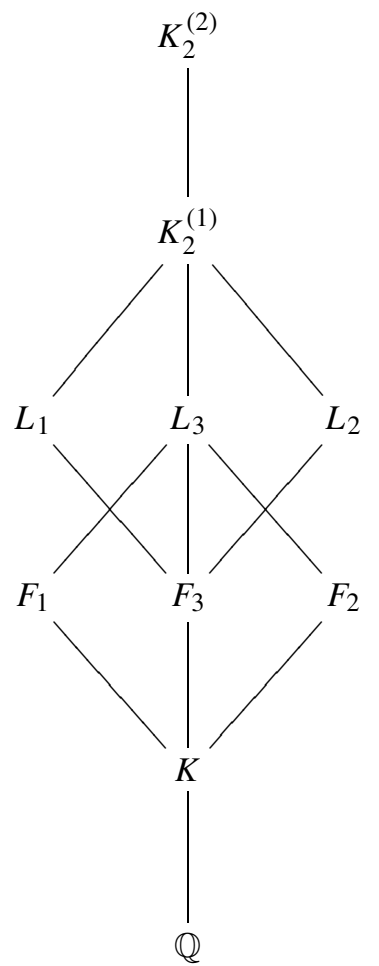

\section{Diagram 1}

where $F_{i}$ 's and $L_{i}$ 's are the extensions over $K$ of degree 2 and 4, respectively.

THEOREM 1. Let the notation be as above. Then the following assertions are equivalent:

1. The group $G$ is non-metacyclic;

2. The 2-class group of $F_{3}$ is of type $(2,2,2)$;

3. The 2-rank of the 2-class group of $F_{3}$ is equal to 3.

PROOF. See [3]. 

remark:

In [3], the authors have proved with the help of the transfer (Verlagerung) the following

REMARK 2.

1. If $G$ is abelian, then four 2-ideal classes of $K$ capitulate in $F_{i}$ for each $i$ and the 2-class group $C_{K, 2}$ of $K$ capitulates in $L_{i}$ for each $i$.

2. If $G$ is non-metacyclic, then the capitulation of 2-ideal classes of $K$ in $F_{3}$ is of type $2 A$ (i.e. two 2-ideal classes of $K$ capitulate in $F_{3}$ and $F_{3} / K$ is of type $(A)$ ).

The aim of this work is the study of the capitulation problem of the 2-ideal classes of an imaginary cyclic quartic number field $K$ with 2-class group of type $(2,4)$, and we determine the structure of $G=\operatorname{Gal}\left(K_{2}^{(2)} / K\right)$. Let $K=k(\sqrt{-n \varepsilon \sqrt{l}})$ with $k=\mathbb{Q}(\sqrt{l})$, $\varepsilon$ the fundamental unit of $k, l$ a prime number and $n$ a square free positive integer prime to $l$. According to E. Brown and C. J. Parry [6] and [7], $C_{K, 2}$ the 2-class group of $K$ is of type $(2,4)$ in the following cases:

1. $l \equiv 5 \bmod 8, n=p \equiv 1 \bmod 4$ and $\left(\frac{p}{l}\right)_{4}=-\left(\frac{l}{p}\right)_{4}=1$, where $p$ is a prime number;

2. $l=2, n=p \equiv 1 \bmod 16$ and $\left(\frac{2}{p}\right)_{4}=-1$, where $p$ is a prime number;

3. $l \equiv 9 \bmod 16, n=1$ and $\left(\frac{2}{l}\right)_{4}=1$.

We denote by $K^{(*)}$ the genus field of $K$. Our two main theorems are the following:

THEOREM A. Let $K=k(\sqrt{-p \varepsilon \sqrt{l}})$ with $k=\mathbb{Q}(\sqrt{l}), \varepsilon$ the fundamental unit of $k, l$ and $p$ two distinct primes satisfying one of the following forms:

1. $l \equiv 5 \bmod 8, p \equiv 1 \bmod 4$ and $\left(\frac{p}{l}\right)_{4}=-\left(\frac{l}{p}\right)_{4}=1$;

2. $l=2, p \equiv 1 \bmod 16$ and $\left(\frac{2}{p}\right)_{4}=-1$.

Then the 2-class field tower of $K$ stops at $K_{2}^{(1)}$, i.e. the group $G$ is abelian. Moreover, four 2-ideal classes of $K$ which capitulate in $F_{i}$ for each $i$ and the 2-class group $C_{K, 2}$ of $K$ capitulates in $L_{i}$ for each $i$.

THEOREM B. Let $K=k(\sqrt{-\varepsilon \sqrt{l}})$ with $k=\mathbb{Q}(\sqrt{l})$, $\varepsilon$ the fundamental unit of $k$, and $l$ a prime number satisfying $l \equiv 9 \bmod 16$ and $\left(\frac{2}{l}\right)_{4}=1$. Then,

1. The group $G$ is non-metacyclic. Moreover, the capitulation of 2-ideal classes of $K$ in $F_{3}=K^{(*)}$ is of type $2 A$;

2. $F_{1}=K(\sqrt{\varepsilon}), F_{2}=K\left(\sqrt{\varepsilon^{\prime}}\right)$ and $L_{3}=K^{(*)}(\sqrt{\varepsilon})$, where $\varepsilon^{\prime}$ denotes the conjugate of $\varepsilon$.

\section{Preliminary results}

This section is reserved for some useful results in the rest of this paper. 
LEMMA 1. Let $K=k(\sqrt{-p \varepsilon \sqrt{l}})$, where $\varepsilon$ is the fundamental unit of $k=\mathbb{Q}(\sqrt{l})$ with $l$ a prime number such that $l=2$ or $l \equiv 5 \bmod 8$ and $p$ a prime number different to $l$ such that $p \equiv 1 \bmod 4$. Then $K^{(*)}=K(\sqrt{p})$.

ProOF. See [2].

LEMma 2. Let $K=k(\sqrt{-\varepsilon \sqrt{l}})$, where $\varepsilon$ is the fundamental unit of $k=\mathbb{Q}(\sqrt{l})$ with $l$ a prime number such that $l \equiv 1 \bmod 8$. Then $K^{(*)}=K(\sqrt{-1})$.

Proof. As $l$ is the unique odd prime of $\mathbb{Q}$ which ramifies in $K$, of ramification index $e_{l}=4$; then, according to [10, Theorem 4, p. 48-49], we have $K^{(*)}=M_{l} K$ where $M_{l}$ is the unique subfield of the $l$-th cyclotomic field of degree $e_{l}=4$. Moreover, it is known that $M_{l}=\mathbb{Q}(\sqrt{\varepsilon \sqrt{l}})$. Thus $K^{(*)}=K(\sqrt{-1})$.

THEOREM 2. Let $p$ and $l$ be two distinct prime numbers such that $l=2$ or $l \equiv$ $1 \bmod 4, p \equiv 1 \bmod 4, h\left(K_{0}\right)($ respectively $h(l p))$ be the class number of $K_{0}=\mathbb{Q}(\sqrt{l}, \sqrt{p})$ (respectively $\mathbb{Q}(\sqrt{l p})$ ) and e be the norm of the fundamental unit of $\mathbb{Q}(\sqrt{l p})$.

1. If $\left(\frac{l}{p}\right)=-1$, then $h\left(K_{0}\right)$ is odd, $h(l p) \equiv 2 \bmod 4$ and $e=-1$.

2. If $\left(\frac{l}{p}\right)=1$, so we have:

(a) If $\left(\frac{l}{p}\right)_{4} \neq\left(\frac{p}{l}\right)_{4}$, then $h\left(K_{0}\right)$ is odd, $h(l p) \equiv 2 \bmod 4$ and $e=1$.

(b) If $\left(\frac{l}{p}\right)_{4}=\left(\frac{p}{l}\right)_{4}=-1$, then $h\left(K_{0}\right)$ is even, $h(l p) \equiv 4 \bmod 8$ and $e=-1$.

(c) If $\left(\frac{l}{p}\right)_{4}=\left(\frac{p}{l}\right)_{4}=1$, then $h\left(K_{0}\right)$ is even and $h(l p) \equiv 0 \bmod 4$. Moreover, if $e=-1$, then $h(l p) \equiv 0 \bmod 8$.

PROOF. See [13].

PROPOSITION 1. Let $L / M$ be a normal biquadratic extension of Galois group of type $(2,2)$. Then $L / M$ has three intermediate fields $N_{1}, N_{2}, N_{3}$ and

$$
h(L)=\frac{2^{d-\kappa-2-v} q(L / M) h\left(N_{1}\right) h\left(N_{2}\right) h\left(N_{3}\right)}{h(M)^{2}},
$$

where $q(L / M)=\left[E_{L}: E_{N_{1}} E_{N_{2}} E_{N_{3}}\right]$ is the unit index of $L / M, d$ is the number of infinite places ramified in $L / M, \kappa$ is the $\mathbb{Z}$-rank of $E_{M}$, and $v=1$ or 0 according to whether $L \subset$ $M\left(\sqrt{E_{M}}\right)$ or not.

PROOF. See [14].

Lemma 3. Let $K=k(\sqrt{-n \varepsilon \sqrt{d}})$ be a cyclic quartic number field, where $\varepsilon$ is the fundamental unit of $k=\mathbb{Q}(\sqrt{d})$ with co-prime square free positive integers $d$ and $n$. Then $\{\varepsilon\}$ is the fundamental system of units of $K$. 
ProOF. See [2].

THEOREM 3. Let $p$ be a prime number such that $p \equiv 1 \bmod 4, K_{0}=\mathbb{Q}(\sqrt{2}, \sqrt{p})$, $\varepsilon_{1}$ (respectively $\left.\varepsilon_{2}, \varepsilon_{3}\right)$ be the fundamental unit of $k_{1}=\mathbb{Q}(\sqrt{2})$ (respectively $k_{2}=\mathbb{Q}(\sqrt{p})$, $\left.k_{3}=\mathbb{Q}(\sqrt{2 p})\right)$ and $F=K_{0}\left(\sqrt{-\varepsilon_{1} \sqrt{2}}\right)$.

1. If $\varepsilon_{3}$ is of norm 1 , then $\left\{\varepsilon_{1}, \varepsilon_{2}, \sqrt{\varepsilon_{3}}\right\}$ is a fundamental system of units of $K_{0}$ and of $F$.

2. Else, $\left\{\sqrt{\varepsilon_{1} \varepsilon_{2} \varepsilon_{3}}, \varepsilon_{2}, \varepsilon_{3}\right\}$ is a fundamental system of units of $K_{0}$ and of $F$.

PROOF. See [1].

THEOREM 4. Let $K_{0}=\mathbb{Q}(\sqrt{l}, \sqrt{p})$ where $p$ and $l$ are two distinct primes such that $l \equiv p \equiv 1 \bmod 4, \varepsilon_{1}$ (respectively $\varepsilon_{2}, \varepsilon_{3}$ ) be the fundamental unit of $k_{1}=\mathbb{Q}(\sqrt{l})$ (respectively $k_{2}=\mathbb{Q}(\sqrt{p}), k_{3}=\mathbb{Q}(\sqrt{l p})$ ) and $F=K_{0}\left(\sqrt{-n \varepsilon_{1} \sqrt{l}}\right)$ where $n$ is a square free positive integer.

1. If $\varepsilon_{3}$ is of norm 1 , then $\left\{\varepsilon_{1}, \varepsilon_{2}, \sqrt{\varepsilon_{3}}\right\}$ is a fundamental system of units of $K_{0}$ and of $F$.

2. Else, $\left\{\sqrt{\varepsilon_{1} \varepsilon_{2} \varepsilon_{3}}, \varepsilon_{2}, \varepsilon_{3}\right\}$ is a fundamental system of units of $K_{0}$ and of $F$.

ProOF. See [1].

\section{Proof of Theorem A}

The proof of Theorem A is based on the following result:

PROPOSITION 2. Let $M$ be a number field with $C_{M, 2}$ the 2-class group of $M$ of type $\left(2^{m}, 2^{n}\right)$. If there is an unramified quadratic extension of $M$ with 2-class number equal to $2^{m+n-1}$; then all the three unramified quadratic extensions of $M$ have 2-class number equal to $2^{m+n-1}$, and the 2-class field tower of $M$ terminates at $M_{2}^{(1)}$.

ProOF. See [5].

In particular, let $K$ be a cyclic quartic number field with $C_{K, 2}$ the 2-class group of $K$ of type $(2,4)$. If there is an unramified quadratic extension of $K$ with 2 -class number equal to 4 , then all the three unramified quadratic extensions of $K$ have 2-class number equal to 4 , and the 2-class field tower of $K$ terminates at $K_{2}^{(1)}$.

THEOREM 5. Let $K=k(\sqrt{-p \varepsilon \sqrt{l}})$ with $k=\mathbb{Q}(\sqrt{l})$, $\varepsilon$ the fundamental unit of $k, l$ and $p$ two distinct primes satisfying one of the following forms:

1. $l \equiv 5 \bmod 8, p \equiv 1 \bmod 4$ and $\left(\frac{p}{l}\right)_{4}=-\left(\frac{l}{p}\right)_{4}=1$;

2. $l=2, p \equiv 1 \bmod 16$ and $\left(\frac{2}{p}\right)_{4}=-1$.

Then $h_{2}\left(K^{(*)}\right)$, the 2-class number of $K^{(*)}$, is equal to 4. 
Proof. By Lemma $1, K^{(*)}=K(\sqrt{p})$. Then $K^{(*)} / k$ is a normal biquadratic extension of Galois group of type $(2,2)$, with quadratic subextensions $K, K^{\prime}=k(\sqrt{-\varepsilon \sqrt{l}})$ and $K_{0}=$ $\mathbb{Q}(\sqrt{l}, \sqrt{p})$. According to Proposition 1 , we have

$$
h_{2}\left(K^{(*)}\right)=\frac{1}{2} q\left(K^{(*)} / k\right) h_{2}(K) h_{2}\left(K^{\prime}\right) h_{2}\left(K_{0}\right),
$$

because $h_{2}(k)=1$ (see [12]), $d=2, \kappa=1$ and $v=0$. As $K_{0} / \mathbb{Q}(\sqrt{l p})$ is an unramified extension and the 2-class group of $\mathbb{Q}(\sqrt{l p})$ is cyclic (see [12]), we have $h_{2}\left(K_{0}\right)=\frac{1}{2} h_{2}(l p)$, where $h_{2}(l p)$ is the 2-class number of $\mathbb{Q}(\sqrt{l p})$. Moreover, $h_{2}(K)=8$ (see [6], [7]) and $h_{2}\left(K^{\prime}\right)=1$ (see [9]), which give $h_{2}\left(K^{(*)}\right)=2 q\left(K^{(*)} / k\right) h_{2}(l p)$. Also we have $\{\varepsilon\}$ is a fundamental system of units of $K$ and of $K^{\prime}$ (Lemma 3), and from the Theorems 2, 3 and 4, $\left\{\varepsilon, \varepsilon_{2}, \sqrt{\varepsilon_{3}}\right\}$ is a fundamental system of units of $K^{(*)}$ and of $K_{0}$, since $\left(\frac{p}{l}\right)_{4} \neq\left(\frac{l}{p}\right)_{4}$ for (1) and $\left(\frac{p}{2}\right)_{4}=(-1)^{\frac{p-1}{8}}=1 \neq\left(\frac{2}{p}\right)_{4}$ for $(2)$, thus $q\left(K^{(*)} / k\right)=1$. Finally, $h_{2}\left(K^{(*)}\right)=2 h_{2}(l p)=4$, where $h_{2}(l p)=2$ (Theorem 2).

3.1. Proof of Theorem A. According to E. Brown and C. J. Parry [6] and [7], $C_{K, 2}$ the 2-class group of $K$ is of type $(2,4)$. By Theorem $5, K^{(*)}$ is the unramified quadratic extension of $K$ with 2-class number equal to 4 . Then all the three unramified quadratic extensions of $K$ have 2-class number equal to 4 , and the 2-class field tower of $K$ terminates with $K_{2}^{(1)}$. On the other hand, by Remark 2, we have four 2-ideal classes of $K$ which capitulate in $F_{i}$ for each $i$ and the 2-class group $C_{K, 2}$ of $K$ capitulates in $L_{i}$ for each $i$. This completes the proof.

EXAmPLE 1. Let $K=\mathbb{Q}(\sqrt{-29 \varepsilon \sqrt{13}})$ where $\varepsilon=\frac{3+\sqrt{13}}{2}$. As $13 \equiv 5 \bmod 8,29 \equiv$ $1 \bmod 4$ and $\left(\frac{29}{13}\right)_{4}=-\left(\frac{13}{29}\right)_{4}=1$, the group $G$ is abelian and $C_{K^{(*)}, 2} \simeq \mathbb{Z} / 2 \mathbb{Z} \times \mathbb{Z} / 2 \mathbb{Z}$.

EXAmple 2. Let $K=\mathbb{Q}(\sqrt{-17 \varepsilon \sqrt{2}})$ where $\varepsilon=1+\sqrt{2}$. As $17 \equiv 1 \bmod 16$ and $\left(\frac{2}{17}\right)_{4}=-1$, the group $G$ is abelian and $C_{K^{(*)}, 2} \simeq \mathbb{Z} / 2 \mathbb{Z} \times \mathbb{Z} / 2 \mathbb{Z}$.

\section{Proof of Theorem B.}

In this section, we want to prove the second main theorem.

LEMMA 4. Let $p$ be a prime number such that $p \equiv 1 \bmod 8$, then

$$
p \neq x^{2}+32 y^{2} \Longleftrightarrow\left(\frac{2}{p}\right)_{4}=-\left(\frac{p}{2}\right)_{4} .
$$

PROOF. See [4].

THEOREM 6. Let $K=k(\sqrt{-\varepsilon \sqrt{l}})$ with $k=\mathbb{Q}(\sqrt{l})$, $\varepsilon$ the fundamental unit of $k$, and $l$ a prime number satisfying $l \equiv 9 \bmod 16$ and $\left(\frac{2}{l}\right)_{4}=1$. Then $h_{2}\left(K^{(*)}\right)$ is equal to $2 h_{2}(-l)$, where $h_{2}(-l)$ is the 2-class number of $\mathbb{Q}(\sqrt{-l})$. Moreover, $h_{2}\left(K^{(*)}\right)=8$. 
Proof. By Lemma $2, K^{(*)}=K(\sqrt{-1})$. So we have that $K^{(*)} / k$ is a normal biquadratic extension of Galois group of type $(2,2)$, with quadratic subextensions $K, L=$ $k(\sqrt{\varepsilon \sqrt{l}})$ and $K_{0}=\mathbb{Q}(\sqrt{l}, \sqrt{-1})$. Therefore, by Proposition 1, we have

$$
h_{2}\left(K^{(*)}\right)=\frac{1}{2} q\left(K^{(*)} / k\right) h_{2}(K) h_{2}(L) h_{2}\left(K_{0}\right),
$$

because $h_{2}(k)=1$ (see [12]), $d=2, \kappa=1$ and $v=0$. As $K_{0} / \mathbb{Q}(\sqrt{-l})$ is an unramified extension and the 2-class group of $\mathbb{Q}(\sqrt{-l})$ is cyclic (see [11]), we have $h_{2}\left(K_{0}\right)=\frac{1}{2} h_{2}(-l)$. Moreover, $h_{2}(K)=8$ (see [7]) and $h_{2}(L)=1$ (see [18]), which give $h_{2}\left(K^{(*)}\right)=2 q\left(K^{(*)} / k\right) h_{2}(-l)$. Also we have $q\left(K^{(*)} / k\right)=1$ (see [16, p.84]). Thus $h_{2}\left(K^{(*)}\right)=2 h_{2}(-l)=8$, since $h_{2}(-l)=4$ and $\left(\frac{l}{2}\right)_{4}=(-1)^{\frac{l-1}{8}}=-1=-\left(\frac{2}{l}\right)_{4}$ (see [4] and Lemma 4).

REMARK 3. The group $G$ is non-abelian because $K_{2}^{(1)} \neq K_{2}^{(2)}$.

4.1. Proof of Theorem B. (1) From the last proof, we see that $K^{(*)}$ is a CM-field with its maximal real subfield $L=k(\sqrt{\varepsilon \sqrt{l}})$ of odd class number (see [18]). Therefore, by [15],

$$
\operatorname{rank} C_{K^{(*)}, 2}=t-1+\operatorname{rank}\left(E_{L} \cap N_{K^{(*)} / L}\left(K^{(*)}\right) / E_{L}^{2}\right),
$$

where $t$ is the number of finite prime ideals ramifying in $K^{(*)} / L$. Using a result in [16], $\left(\frac{\varepsilon \sqrt{l}}{2_{1}}\right)=\left(\frac{\varepsilon \sqrt{l}}{2_{2}}\right)=\left(\frac{2}{l}\right)_{4}=1$ where $2_{1}$ and $2_{2}$ are the prime ideals in $k$ above 2 , we see that 2 splits completely in $L$. Thus exactly 4 prime ideals are ramified in $K^{(*)} / L$. It follows from $\operatorname{rank}\left(E_{L} \cap N_{K^{(*)} / L}\left(K^{(*)}\right) / E_{L}^{2}\right)=0$ (see [16, p.84]) that rank $C_{K^{(*)}, 2}=3$. Further $h_{2}\left(K^{(*)}\right)=8$ by Theorem 6 . Then, since $C_{K^{(*)}, 2}$ is of type $(2,2,2)$, we have $F_{3}=K^{(*)}$. On the other hand , $C_{K, 2}$ is of type $(2,4)$ by Brown and Parry [7], while $G$ is non-metacyclic by Theorem 1 . Combining them, we can use Remark 2 to see that the capitulation of 2 -ideal classes of $K$ in $K^{(*)}$ is of type $2 A$.

(2) We know by Cohn [8], that if $l \equiv 1 \bmod 8$, then $F=K_{0}(\sqrt{\varepsilon})$ is an unramified quadratic extension over $K_{0}$ where $K_{0}=\mathbb{Q}(\sqrt{l}, \sqrt{-1})$. It is easy to see that $\sqrt{\varepsilon} \notin F_{3}$. Therefore $K F=F_{3}(\sqrt{\varepsilon})$ is an unramified quadratic extension over $K K_{0}=F_{3}$. 


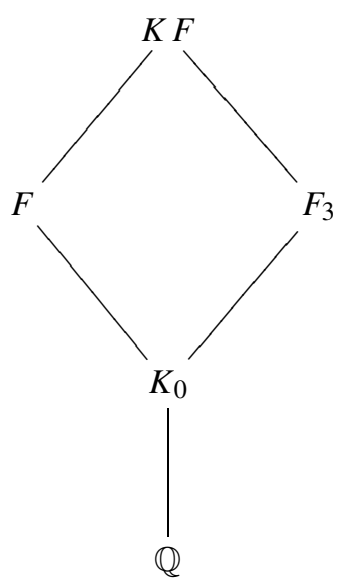

\section{Diagram 2}

Moreover, we have that $F_{3}$ is unramified over $K$. Thus $K F$ is unramified over $K$ and the Galois group of $K F / K$ is of type $(2,2)$. Consequently, $F_{1}=K(\sqrt{\varepsilon}), F_{2}=K\left(\sqrt{\varepsilon^{\prime}}\right)$ and $L_{3}=K^{(*)}(\sqrt{\varepsilon})$.

EXAMPLE 3. Let $K=\mathbb{Q}(\sqrt{-\varepsilon \sqrt{73}})$ where $\varepsilon=1068+125 \sqrt{73}$. As $73 \equiv 9 \bmod 16$ and $\left(\frac{2}{73}\right)_{4}=1$, the group $G$ is non-metacyclic, $C_{K^{(*)}, 2} \simeq \mathbb{Z} / 2 \mathbb{Z} \times \mathbb{Z} / 2 \mathbb{Z} \times \mathbb{Z} / 2 \mathbb{Z}$ and $K^{(*)} / K$ is of type $2 A$ where $K^{(*)}=K(\sqrt{-1})$.

ACKNOWLEDGment. We would like to thank the referees for the helpful and constructive comments.

\section{References}

[1] A. AZIZI and M. TALBI, Capitulation des 2-classes d'idéaux de certains corps biquadratiques cycliques, Acta Arithmetica 127 (2007), 231-248.

[2] A. AZIZI and M. TALBI, Capitulation dans certaines extensions non ramifiées de corps quartiques cycliques, Archivum Mathematicum (Brno), Tomus 44 (2008), 271-284.

[ 3 ] A. AZIZI and M. TAous, Capitulation des 2-classes d'idéaux de type (2, 4), arXiv 30 Jan 2014.

[4] P. BARRUCAND and H. COHN, Note on primes of type $x^{2}+32 y^{2}$, class number and residuacity, J. Reine Angew. Math. 238 (1969), 67-70.

[ 5 ] E. BENJAMin, F. LEMMERMEYER and C. SNYDER, Real quadratic fields with abelian 2-class field tower, J. Number Theory 73 (1998), 182-194.

[ 6 ] E. BROWN and C. J. PARRY, The 2-class group of certain biquadratic number fields I, J. Reine Angew. Math. 295 (1977), 61-71.

[ 7 ] E. BROWN and C. J. PARRY, The 2-class group of certain biquadratic number fields II, Pacific J. Math. 78, No. 1, (1978), 11-26. 
[ 8 ] H. Cohn, The explicit Hilbert 2-cyclic class fields of $\mathbb{Q}(\sqrt{-p})$, J. Reine Angew. Math. 321 (1981), 64-77.

[ 9 ] P. E. Conner and J. Hurrelbrink, Class Number Parity, Series in Pure Mathematic, World Scientific, 1988.

[10] M. IsHIDA, The genus fields of algebraic number fields, Lecture notes in mathematics 555, Springer-Verlag, 1976.

[11] P. KAPLAN, Divisibilité par 8 du nombre des classes des corps quadratiques dont le 2-groupe des classes est cyclique, et réciprocité biquadratique, J. Math. Soc. Japan 25, No. 4, (1973), 596-608.

[12] P. Kaplan, Sur le 2-groupe des classes d'idéaux des corps quadratiques, J. Reine Angew. Math. 283/284 (1976), 313-363.

[13] R. KUČERA, On the parity of the class number of biquadratic field, J. Number Theory $\mathbf{5 2}$ (1995), 43-52.

[14] F. LEMMERMEYER, Kuroda's class number formula, Acta Arith. 66(3) (1994), 245-260.

[15] F. LEMMERMEYER, On the 2-class field tower of imaginary quadratic number fields, J. Théorie des Nombres de Bordeaux 6 (1994), 261-272.

[16] M. TAlBI, Capitulation des 2-classes d'idéaux de certains corps quartiques de type $(2,2)$, thèse. Université Mohamed Premier. Oujda, 2008.

[17] O. Tauss Ky, A Remark on the Class Field Tower, J. London Math. Soc. 12 (1937), 82-85.

[18] L. C. WAShington, Introduction to cyclotomic fields, Graduate texts in mathematics 83 (1996).

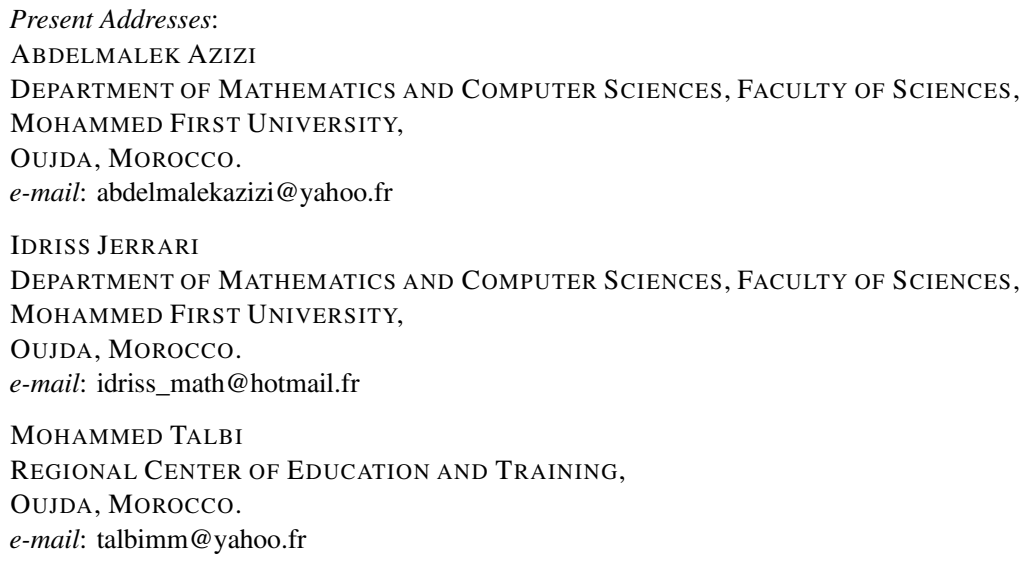

